\title{
Neural Alpha Dynamics in Younger and Older Listeners Reflect Acoustic Challenges and Predictive Benefits
}

\author{
Malte Wöstmann, ${ }^{1,2}$ Björn Herrmann, ${ }^{1}$ Anna Wilsch, ${ }^{1}$ and ${ }^{\top}$ Jonas Obleser ${ }^{1}$ \\ ${ }^{1}$ Max Planck Research Group “Auditory Cognition,” Max Planck Institute for Human Cognitive and Brain Sciences, 04103 Leipzig, Germany, and \\ ${ }^{2}$ International Max Planck Research School on Neuroscience of Communication, 04103 Leipzig, Germany
}

\begin{abstract}
Speech comprehension in multitalker situations is a notorious real-life challenge, particularly for older listeners. Younger listeners exploit stimulus-inherent acoustic detail, but are they also actively predicting upcoming information? And further, how do older listeners deal with acoustic and predictive information? To understand the neural dynamics of listening difficulties and according listening strategies, we contrasted neural responses in the alpha-band $(\sim 10 \mathrm{~Hz})$ in younger $(20-30$ years, $n=18)$ and healthy older $(60-70$ years, $n=20)$ participants under changing task demands in a two-talker paradigm. Electroencephalograms were recorded while humans listened to two spoken digits against a distracting talker and decided whether the second digit was smaller or larger. Acoustic detail (temporal fine structure) and predictiveness (the degree to which the first digit predicted the second) varied orthogonally. Alpha power at widespread scalp sites decreased with increasing acoustic detail (during target digit presentation) but also with increasing predictiveness (in-between target digits). For older compared with younger listeners, acoustic detail had a stronger impact on task performance and alpha power modulation. This suggests that alpha dynamics plays an important role in the changes in listening behavior that occur with age. Last, alpha power variations resulting from stimulus manipulations (of acoustic detail and predictiveness) as well as task-independent overall alpha power were related to subjective listening effort. The present data show that alpha dynamics is a promising neural marker of individual difficulties as well as age-related changes in sensation, perception, and comprehension in complex communication situations.
\end{abstract}

Key words: acoustic detail; ageing; alpha oscillations; prediction; speech

\section{Introduction}

Natural environments are rich of sensory information from both relevant (i.e., target) and irrelevant (i.e., noise) sources. Selective attention to relevant information enhances the neural representation of targets (Desimone and Duncan, 1995). According to the "functional inhibition" framework, neural alpha oscillations $(\sim 10 \mathrm{~Hz})$ support target processing through the inhibition of task-irrelevant sensory modalities or brain processes (Jensen and Mazaheri, 2010). Alpha power is modulated by task-irrelevant sensory interference (Sauseng et al., 2009), by anticipation of distracting interference (e.g., Bonnefond and Jensen, 2012), and by predictions about nondistracting events (e.g., van Ede et al., 2011). The specific role of alpha oscillations in attentional processing is indicated, for example, by alpha power increase in parieto-occipital regions when attention shifts toward the audi-

\footnotetext{
Received Aug. 6, 2014; revised Nov. 28, 2014; accepted Dec. 3, 2014.

Author contributions: M.W. and J.0. designed research; M.W. performed research; M.W., B.H., A.W., and J.0. analyzed data; M.W., B.H., A.W., and J.O. wrote the paper.

This work was supported by Max Planck Society Max Planck Research Group Grant to J.0. We thank Dunja Kunke for help during data acquisition and two anonymous reviewers who were particularly helpful in improving the manuscript.

The authors declare no competing financial interests.

Correspondence should be addressed to either Malte Wöstmann or Jonas Obleser, Max Planck Research Group

"Auditory Cognition," Max Planck Institute for Human Cognitive and Brain Sciences, Stephanstraße 1a, 04103 Leipzig, Germany. E-mail:woestmann@cbs.mpg.de. or obleser@cbs.mpg.de.

DOI:10.1523/JNEUROSCI.3250-14.2015

Copyright $\odot 2015$ the authors $\quad 0270-6474 / 15 / 351458-10 \$ 15.00 / 0$
}

tory modality (Adrian, 1944; Foxe et al., 1998; Mazaheri et al., 2014).

Speech perception against competing talkers is a paradigmatic example for distracting interference, but the role of alpha oscillations in these situations is weakly explored (Kerlin et al., 2010; Strauß et al., 2014). Alpha power increases if listening conditions become more demanding due to degradation of acoustic detail (Obleser et al., 2012; Becker et al., 2013), increasing syntactic complexity (Meyer et al., 2013) and low temporal expectancy (Wilsch et al., 2014). However, it is thus far unknown whether cues that allow listeners to predict upcoming information impinge upon alpha oscillations in a similar manner. Moreover, it is unclear whether listeners of different age use acoustic information and predictive cues similarly to overcome listening challenges in multitalker situations.

For healthy older adults, listening in multitalker situations is particularly effortful (Pichora-Fuller and Souza, 2003). This effort cannot be explained by sensory hearing loss alone (Wingfield et al., 2005; Meister et al., 2012). Instead, attentional control changes with age (Tun et al., 2002; Gazzaley et al., 2005), which might involve that older listeners strongly attend to acoustic features of the stimulation (e.g., Passow et al., 2014) and that they are unable to ignore task-irrelevant acoustic stimuli (e.g., Chao and Knight, 1997). Importantly, age differences in neural responses might be driven by reduced sensory acuity in the elderly (Peelle et al., 2011) and therefore need rigorous experimental control. In the present study, stimulus intensities were individu- 
A Trial design for the auditory number comparison task
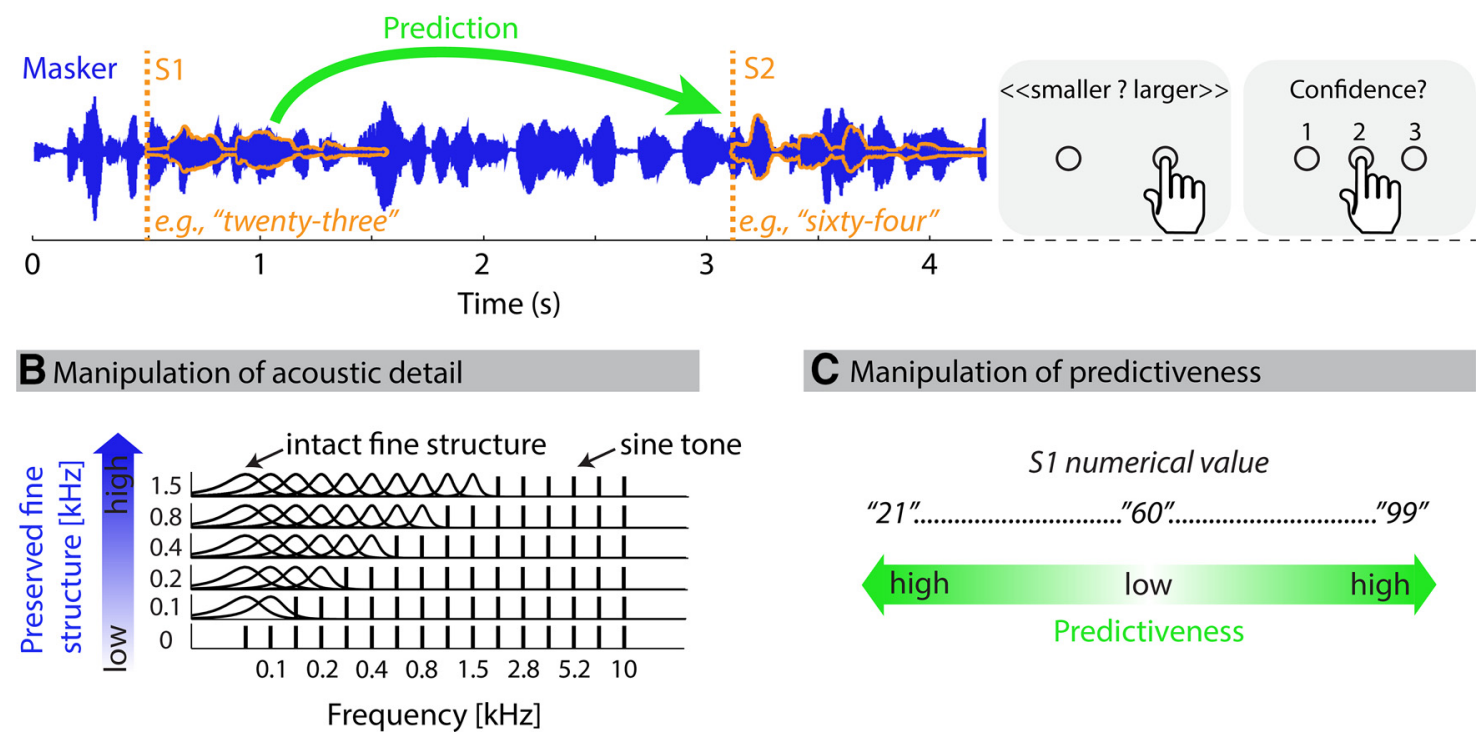

Manipulation of predictiveness

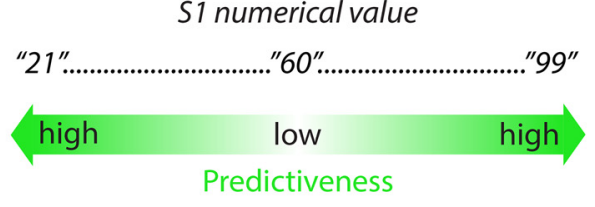

Figure 1. Trial design and experimental manipulation. $A, 0$ n each trial, participants listened to two spoken digits ( $S 1$ and $S 2$, orange) embedded in a distracting speech masker. Their task was to indicate whether $\mathrm{S} 2$ was smaller or larger than $\mathrm{S1}$, and how confident they were in this decision. $\boldsymbol{B}$, Acoustic detail was varied over six levels by parametrically preserving temporal fine structure of the signal's low frequencies (blue color gradient). $C$, The degree to which $\$ 1$ was predictive of the numerical value of $\$ 2$ was operationalized as the numerical distance between $\$ 1$ and the midpoint of all possible numbers (60; green color gradient).

ally adjusted for hearing acuity (frequency-specific adjustments to individual audiograms) and for speech-in-noise thresholds, to exclude "trivial" age effects in oscillatory alpha band dynamics related to decreasing stimulus audibility at an older age.

Participants performed a numerical comparison (Moyer and Landauer, 1967), where two spoken digits were embedded in a continuous stream of distracting speech. Acoustic detail (temporal fine structure) (Moore, 2008) and the degree to which the first digit predicted the second (Scheibe et al., 2010) varied orthogonally. Here we show that both stimulus manipulations (acoustic detail and predictiveness) modulate alpha power. We further provide evidence that these effects relate to subjective listening effort. Critically, aging affected behavioral performance as well as alpha power modulations by acoustic detail, suggesting that alpha power dynamics track age-related changes of listening behavior in challenging acoustic environments.

\section{Materials and Methods}

Participants. Eighteen younger (mean age: 25.6 years; age range: $20-30$ years; 9 females) and 20 older (mean age: 64.0 years; age range: $60-70$ years; 11 females) healthy, right-handed German native speakers participated in the experiment. Data from two additional younger participants were recorded but excluded from the analysis because of technical problems during recording and overall below-chance task performance. Participants gave informed consent and were financially compensated for their participation. Procedures were in accordance with the Declaration of Helsinki and approved by the local ethics committee of the University of Leipzig Medical faculty.

Hearing acuity. Participants' pure-tone air-conduction audiometric thresholds (at frequencies of $0.25,0.5,1,2,3,4,6$, and $8 \mathrm{kHz}$ ) were assessed by a trained audiologist separately for both ears in steps of $5 \mathrm{~dB}$ hearing level using a clinical audiometer (according to standardized procedures described by British Society of Audiology, 2011). Participants did not show interaural asymmetries ( $\geq 20 \mathrm{~dB}$ difference between both ears at more than two frequencies). Individual audiograms were used for frequency-specific adjustment of stimulus intensities (see below). Participants' audiograms, details concerning the individual stimulus adjust- ments, and the analysis of the event-related potential for the same dataset have been published previously (Wöstmann et al., 2014).

Number comparison task. Participants performed an auditory version of a number comparison task (Moyer and Landauer, 1967). In detail, each trial started with the visual presentation of the two response options ("kleiner," "größer"; German for "smaller" and "larger," respectively) on the computer screen, followed (after $1.5 \mathrm{~s}$ ) by the binaural presentation of a continuous speech masker and two sequentially presented spoken digits (Fig. 1A). Following sound offset, participants indicated via button press on a response box whether the second digit was smaller (left button pressed with left thumb) or larger (right button pressed with right thumb) than the first. Subsequently, they rated their confidence in this decision on a 3 point scale $(1$, unconfident; 3 , confident). Participants were instructed to perform the number comparison as fast and as accurately as possible. The next trial started self-paced with an additional button press. To eliminate possible effects of participants' eye closure on alpha oscillations, participants were instructed to keep their eyes open during the trials. Participants were monitored via video camera to make sure that they did not close their eyes during acoustic stimulation. Stimulation was controlled by Presentation software (Neurobehavioral Systems).

Speech materials. German spoken digits ranging from 21 to 99 (excluding multiples of 10) were recorded from a trained female speaker (sampling rate, $44.1 \mathrm{kHz}$ ). All digits contained four syllables (mean digit length \pm SEM: $1.125 \pm 0.007 \mathrm{~s}$ ). A distracting masker stimulus was extracted from a German audiobook (Oscar Wilde, The young king, German title: Der junge König) spoken by a different female talker (sampling rate, $44.1 \mathrm{kHz}$ ). To increase the energetic overlap of masker and digits, silent periods $>70 \mathrm{~ms}$ were removed automatically from the masker (using a customized MATLAB script R2013a; MathWorks). The resulting masker stimulus had a length of $\sim 30 \mathrm{~min}$, from which we extracted 1000 random snippets.

For each experimental stimulus, two different target digits (referred to as S1 and S2) and one masker snippet (referred to as masker) were selected randomly. Digits and masker were combined by adding the waveforms such that S1 and S2 were presented $0.5 \mathrm{~s}$ and $3.125 \mathrm{~s}$ after masker onset, respectively. The interval between S1 offset and S2 onset was on 
average $1.5 \mathrm{~s}$ (depending on S1 duration). Stimuli ended with S2 offset and had an average duration of $\sim 4.25 \mathrm{~s}$ (Fig. $1 A$ ).

Experimental conditions. In the current study, stimuli were manipulated along two orthogonal dimensions: acoustic detail and predictiveness. For the acoustic detail manipulation, the temporal fine structure of the combined signal (composed of masker and digits) was manipulated. In detail, the signal was divided into 16 overlapping frequency channels using a gammatone filterbank (implemented in the auditory toolbox for MATLAB) (Slaney, 1993). Channel center frequencies increased exponentially from 0.08 to $10 \mathrm{kHz}$. Six temporal fine structure conditions comprising different levels of acoustic degradation were generated. For a particular condition, frequency channels above one of six fine structure cutoffs $(0,0.11,0.21,0.4,0.76$, and $1.45 \mathrm{kHz})$ were degraded, whereas channels below and including the cutoff were left unchanged (Hopkins et al., 2008) (Fig. 1B). In channels above the cutoff, the speech envelope was extracted using the Hilbert transform (Smith et al., 2002). The envelope was used to modulate a sinusoidal tone with random starting phase at the channel's center frequency. The resulting signal was filtered again with the initial gammatone filters to remove out-of-channel frequency components (Lunner et al., 2012). Finally, intact and modified channels were combined, yielding six different levels of temporal fine structure preservation. In sum, this manipulation of the temporal fine structure degraded fast spectrotemporal fluctuations, rendering the perceptual segregation of digits and masker more demanding. Slow temporal envelope fluctuations were left intact (Shamma and Lorenzi, 2013). Critically, degraded stimuli were intelligible as the number of frequency channels (16) was sufficiently high (Shannon et al., 1995; Obleser et al., 2007, 2008).

For the predictiveness manipulation, the degree to which the S1 digit was predictive of the S2 digit was operationalized as the numerical distance between $\mathrm{S} 1$ and the midpoint of all possible digits. In detail, digits in the experiment ranged between 21 and 99, meaning that 60 was the midpoint of all digits. When the $\mathrm{S} 1$ digit was considerably $<60$, participants could predict that the S2 digit would likely be larger than S1, and vice versa for $S 1$ digits $>60$. Contrary, if the $S 1$ digit was close to 60 , no prediction about whether $\mathrm{S} 2$ would be smaller or larger could be made. Thus, with increasing numerical distance between $S 1$ and 60, participants could better predict whether $\mathrm{S} 2$ would be smaller or larger (Fig. 1C).

Individual stimulus adjustments. Before the actual experiment, stimuli underwent a frequency-specific amplification (CAMEQ) (Moore et al., 1998) to account for considerable differences in hearing thresholds estimated in the audiograms, especially between age groups. This procedure aimed at the same overall perceived stimulus loudness for all participants corresponding to a stimulus intensity of $\sim 75 \mathrm{~dB}$ SPL for a listener with average normal hearing (audiometric thresholds of $0 \mathrm{~dB}$ hearing level at all test frequencies).

Because speech-in-noise hearing thresholds cannot be matched between age groups by controlling only for pure-tone audiometric thresholds (see Pichora-Fuller et al., 1995), an additional adaptive tracking procedure (Levitt, 1971) was used to estimate the digit-to-masker soundlevel ratio yielding $70.9 \%$ correct responses in our number comparison task under the most extreme acoustic degradation $(0 \mathrm{kHz}$ fine structure cutoff). To this end, the sound level of the digits was adapted while keeping the masker sound level fixed at $-30 \mathrm{~dB}$ full-scale (root mean square). Mean digit-to-masker sound-level ratio for young participants was $-20.83 \mathrm{~dB}( \pm 0.72 \mathrm{SEM})$ and for older participants $-15.35 \mathrm{~dB}$ $( \pm 1.29 \mathrm{SEM})$, which were significantly different $\left(t_{36}=3.60 ; p=0.001\right.$; $r=0.51)$.

Procedure. After the individual stimulus adjustments were applied, participants took part in the main experiment. Acoustic stimulation and EEG recording were performed in an electrically shielded and soundattenuated booth. Participants were seated in a comfortable chair in front of a computer screen. Auditory stimuli were presented via TDH39 audiometric headphones. Each participant performed 300 trials, 50 for each temporal fine structure cutoff level. The experiment was divided into five blocks. Each block contained 10 trials of each fine structure cutoff in random order. Predictiveness of the second digit was fully randomized across the 300 trials. That is, the numerical values of S1 and S2 varied randomly across trials with the constraint that in half of the trials S2 was larger than S1 and in the other half smaller than S1 (S1 and S2 digits were never equal). The experiment lasted $\sim 70 \mathrm{~min}$.

Statistical analysis of behavioral data. Participants' performance in the auditory number comparison task was quantified using weighted percentage correct responses (weighted accuracy). In detail, the binary response in each trial (correct vs incorrect) was weighted by the trial's confidence rating to get a more fine grained (six-level) measure of task performance (see also Kitayama, 1991; Herrmann et al., 2014). To this end, a correct response was transformed to $100 \%$ weighted accuracy in case of a high confidence rating, to $80 \%$ in case of medium confidence, and to $60 \%$ in case of low confidence. Similarly, an incorrect response was transformed to $40 \%$ weighted accuracy for a low confidence rating, to $20 \%$ for medium confidence, and to $0 \%$ for high confidence. In the remainder of this paper, we use, for simplicity, the term "accuracy" to refer to accuracy weighted by confidence ratings.

As a second performance measure, we quantified participants' response times in the number comparison task. Response times corresponded to the time interval between the onset of the second digit and participants' button press to indicate whether the second digit was smaller or larger than the first.

Changes in behavioral performance (accuracy and response times) as a function of acoustic detail were tested as follows: For each temporal fine structure cutoff level, single-trial accuracy values and response times were averaged (ignoring predictiveness). For each participant, a linear function was fitted to the averaged accuracy values and response times as a function of six linearly spaced fine structure cutoffs (predictor values: $-2.5,-1.5,-0.5,0.5,1.5,2.5)$. Linear spacing of cutoffs for fitting was used because logarithmic spacing of physical stimulus frequencies relates to linear spacing in auditory perception (Attneave and Olson, 1971). The estimated linear coefficients were subsequently tested against zero using one-sample $t$ tests. Significant differences from zero would indicate modulation of behavioral performance by acoustic detail.

Changes in behavioral performance (accuracy and response times) as a function of predictiveness were examined as follows: The degree to which $\mathrm{S} 1$ was predictive of $\mathrm{S} 2$ on each trial was quantified as the absolute numerical difference between $\mathrm{S} 1$ and 60 (average digit across the experiment). Predictiveness values across all trials were divided into six percentile bins (no overlap), and single-trial accuracy values and response times were averaged within each bin. Linear functions were fitted to the averaged accuracy values and response times for each participant as a function of percentile bins (zero-centered predictor values: $-2.5,-1.5$, $-0.5,0.5,1.5,2.5)$. The estimated linear coefficients were tested against zero using one-sample $t$ tests. Significant differences from zero would indicate modulation of behavioral performance by predictiveness.

To test for differences between performance modulation by acoustic detail and predictiveness as well as for differences between age groups, repeated-measures ANOVAs were performed. The within-subject factor was stimulus dimension (acoustic detail vs predictiveness) and the between-subject factor age group (younger vs older) using the estimated linear coefficients for accuracy and response times as dependent measures. To follow up on significant age group $\times$ stimulus dimension interactions, post hoc independent-samples $t$ tests were used to test for effects of age group on linear coefficients separately for the manipulation of acoustic detail and predictiveness.

Overall performance between age groups was compared by submitting participants' average accuracy and average response times (across all manipulation levels) to independent-samples $t$ tests.

EEG recording. EEGs were recorded at a $500 \mathrm{~Hz}$ sampling rate with a DC $-135 \mathrm{~Hz}$ filter pass band (TMS International). Twenty-eight electrodes $(\mathrm{Ag} / \mathrm{Ag}-\mathrm{Cl})$ were placed at the following positions (Easycap): $\mathrm{Fpz}$, Fp1, Fp2, Fz, F3, F4, F7, F8, FC3, FC4, FT7, FT8, Cz, C3, C4, T7, T8, CP5, $\mathrm{CP} 6, \mathrm{Pz}, \mathrm{P} 3, \mathrm{P} 4, \mathrm{P} 7, \mathrm{P} 8, \mathrm{O} 1, \mathrm{O}_{2}$, left mastoid (A1), and right mastoid (A2). The reference electrode was placed at the tip of the nose and the ground electrode at the sternum. The electro-oculogram was recorded from vertical and horizontal bipolar montages. All electrode resistances were kept $<5 \mathrm{k} \Omega$.

Data were analyzed offline using custom MATLAB scripts and the fieldtrip toolbox (version 2013-01-14) (Oostenveld et al., 2011). Epochs were extracted from the continuous signal time-locked to masker onset 
( -1.5 to $5.5 \mathrm{~s})$. Epochs were low-pass filtered at $100 \mathrm{~Hz}$. An independent components analysis was performed on the epoched data. Components corresponding to eye blinks, saccadic eye movements, muscle activity, electrode drifts, and heartbeats were identified and rejected by inspection of the components' topographies, frequency spectra, and time courses. Remaining artifact-contaminated trials were deleted after visual inspection of EEG waveforms at all electrodes. On average, $7 \pm 1 \%$ (SEM) trials in each participant were rejected from further analyses.

Time-frequency representations of single trials were estimated by convolving the single-trial time series with a family of Morlet wavelets between 1 and $30 \mathrm{~Hz}$ (in steps of $0.5 \mathrm{~Hz}$; width: 7 cycles) and from -1.5 to $5.5 \mathrm{~s}$ (in steps $0.02 \mathrm{~s}$ ). Single-trial power was obtained by squaring the magnitude of the estimated complex wavelet transform coefficients. Power changes relative to a prestimulus baseline were computed by means of subtraction and division by the average power from -0.8 to $0 \mathrm{~s}$ (relative change baseline).

Overall temporal dynamics of alpha power. We analyzed the overall time course of alpha power during the number comparison task, irrelevant of varying acoustic detail and stimulus predictiveness (see Fig. 3). To this end, single-trial oscillatory power was averaged across all conditions, frequency bins in the alpha band $(7-13 \mathrm{~Hz})$, and five parietal electrodes exhibiting the strongest alpha power (CP5, P3, Pz, P4, CP6; see Fig. 3A). To test for age effects, time courses of alpha power were compared between age groups by contrasting average alpha power estimates in steps of $0.02 \mathrm{~s}$ with independent-samples $t$ tests. $p$ values from multiple $t$ tests were adjusted to control the false discovery rate (FDR) (Benjamini and Hochberg, 1995).

Effects of experimental manipulations on EEG data. Modulatory influences of acoustic detail and predictiveness on oscillatory power were analyzed as follows. For each participant, two linear functions were fitted to single-trial power values (independently for each time-frequency bin and electrode): (1) as a function of acoustic detail; and (2) as a function of predictiveness percentile bins (using parametric regression $t$ tests for independent samples implemented in the $f t$ freqstatistics function in fieldtrip; predictor values: $-2.5,-1.5,-0.5,0.5,1.5,2.5)$. This resulted in one time-frequency-electrode matrix of estimated linear coefficients for the acoustic detail manipulation and in one matrix for the predictiveness manipulation, reflecting the modulation of single-trial power for each participant.

For the statistical analysis across participants, we focused on the alpha frequency range $(\sim 10 \mathrm{~Hz})$ for which we hypothesized to observe power changes due to manipulations of acoustic detail and predictiveness (see Introduction). Furthermore, analyses were conducted including participants of both age groups, followed by analyses of age differences where effects for all participants (younger and older) were significant. To this end, estimated linear coefficients in the $7-13 \mathrm{~Hz}$ frequency band, the $0-5.2 \mathrm{~s}$ time window, and all scalp electrodes were tested against zero using two cluster-based permutation one-sample $t$ tests (Maris and Oostenveld, 2007): one for the effect of acoustic detail and one for the effect of predicitveness. These tests clustered $t$ values of adjacent bins in timefrequency-electrode space with a $p$ value $<0.05$, considering a minimum of three neighboring electrodes as a cluster. The summed $t$ value of each cluster was computed and compared against the distribution of 1000 iteratively and randomly drawn clusters from data for which condition labels were permuted. The cluster $p$ value resulted from the proportion of Monte Carlo iterations in which the summed $t$-statistic of the observed cluster was exceeded. As we performed this analysis as a two-sided test (for clusters exhibiting positive and negative effects), clusters with $p<$ 0.025 were considered significant. This analysis revealed four significant clusters: two for the effect of acoustic detail and two for the effect of predictiveness (see Fig. 4).

To test for an effect of age group on linear coefficients in all four clusters, a repeated-measures ANOVA (within-subject factor: cluster; between-subject factor: age group) was calculated for the averaged linear coefficients in the four clusters. Because task-related power suppression is known to depend on overall power (Doppelmayr et al., 1998; Klimesch et al., 2003), we controlled for effects of overall alpha power and the decrease in alpha power over the trial time course (see Fig. 3 ) in two additional ANOVAs: For the first additional ANOVA, we extracted over- all alpha power (averaged across conditions) at those time-frequencyelectrode bins of the four significant clusters, resulting in four covariates that were included in the repeated-measures ANOVA. For the second additional ANOVA, linear coefficients estimated from linear fits to the overall alpha power (averaged across $7-13 \mathrm{~Hz}$ and electrodes CP5, $\mathrm{P} 3, \mathrm{Pz}, \mathrm{P} 4, \mathrm{CP} 6$ ) as a function of time (ranging from $0.82 \mathrm{~s}$ to $4.88 \mathrm{~s}$, that is from cluster A1 to A2) were included as a covariate. To follow up a significant age group $\times$ cluster interaction, post hoc independent-samples $t$ tests were used to test for effects of age group in each cluster.

To test for a possible interaction between acoustic detail and predictiveness on alpha power modulation, we averaged alpha power estimates of only those time-frequency-electrode power bins that had been part of both the acoustic detail and the predictiveness clusters in the analyses outlined above (Obleser et al., 2012). These average power estimates were submitted to a repeated-measures ANOVA (within-subject factors: acoustic detail, predictiveness; between-subject factor: age group).

Effect sizes. To estimate effect sizes for $F$ statistics (ANOVAs), we calculated the partial $\eta$-squared $\left(\eta_{\mathrm{p}}^{2}\right)$. For $t$-statistics (dependent and independent-samples $t$ tests), we estimated the effect size measure $r$, which is bound between 0 and 1 (Rosenthal, 1994). Effect sizes for multiple $t$ tests (e.g., for all time-frequency-electrode bins belonging to a significant cluster) were estimated by averaging $r$ values across individual tests into a composite cluster-effect size $R$.

The relation between alpha oscillations and subjective difficulty measures. We further tested whether alpha power modulations (by acoustic detail and predictiveness) within observed clusters were related to participants' subjective listening effort in background noise and confidence ratings. In detail, alpha power modulation was quantified as the average of the linear coefficients across significant time points, frequency bins, and electrodes as well as across the four significant clusters of acoustic detail and predictiveness. Subjective listening effort in background noise was quantified as the response in a post experiment inquiry where participants answered the question, "In general, how difficult is it for you to listen to a single speaker if several other people are talking loudly in the background?" on a 5-point Likert scale (1, easy; 5, difficult; question translated from German). The Spearman correlation was obtained between ratings of subjective listening effort in noise and alpha power modulation (see Fig. 5A).

To test for an impact of alpha power on participants' subjectively experienced certainty in the numerical decision, we analyzed whether alpha power in trials with the same level of acoustic detail and predictiveness would affect participants' confidence ratings. To this end, we averaged participants' single-trial alpha power $(7-13 \mathrm{~Hz})$ across all scalp electrodes and in the time period of significant alpha power modulations, that is, between the onset of the earliest significant cluster $(0.82 \mathrm{~s})$ and the offset of the latest significant cluster $(4.88 \mathrm{~s})$. Subsequently, within each combination of the 6 (acoustic detail) $\times 6$ (predictiveness) levels, trials were divided into three (nonoverlapping) percentile bins based on alpha power. In detail, trials were assigned to low, medium, or high alpha power, depending on whether their alpha power was among the lowest third $(0 \%-33 \%)$, medium third $(33 \%-66 \%)$, or highest third $(66 \%-$ $100 \%)$ out of all trials within a particular acoustic detail-predictiveness combination. Next, the average over the trial's confidence ratings in these three percentiles was calculated. Critically, the sorting of single trials according to alpha power was performed independently for each combination of acoustic detail and predictiveness, and average confidence ratings were thus independent of between-condition effects. For each participant, mean confidence ratings were subsequently averaged over all combinations of acoustic detail and predictiveness levels, to obtain three confidence values for low, medium, and high alpha power trials, respectively. For each participant, a linear function was fitted to confidence values as a function of alpha power bin (predictor values: $-1,0,1$ ). Estimated linear coefficients across participants were tested against zero using a one-sample $t$ test. A significant difference from zero would indicate a modulation of confidence ratings by alpha power. Linear coefficients of younger and older participants were compared using an independent-samples $t$ test (see Fig. $5 B$ ). 

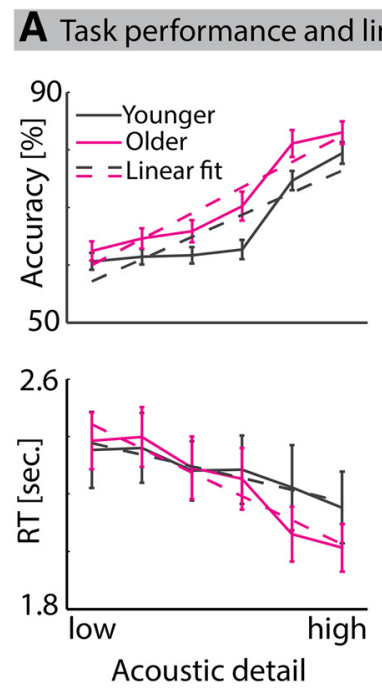
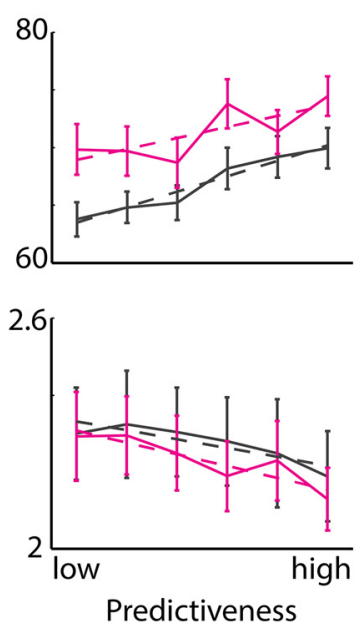

B Linear coefficients

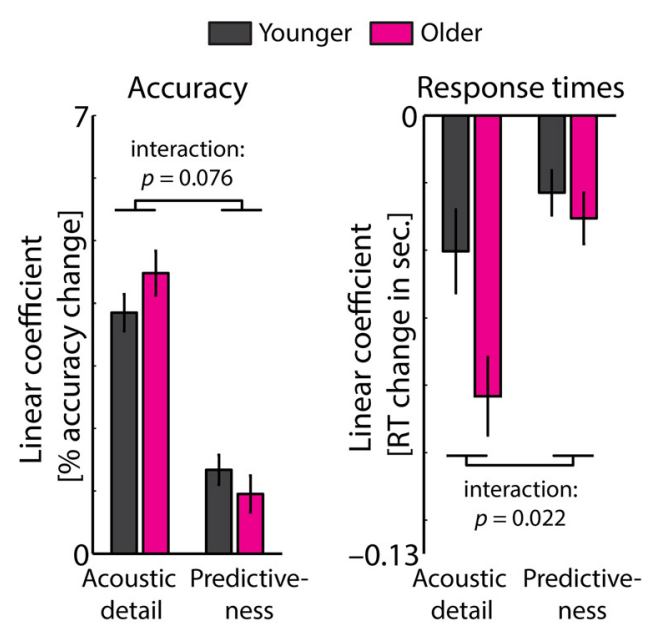

Figure 2. Effects of acoustic detail and predictiveness on task performance. $\boldsymbol{A}$, Mean accuracy (top row) and response times (RT; bottom row) as a function of acoustic detail (left column) and predictiveness (right column). Solid lines indicate average accuracy and RTs for younger (black) and older (magenta) participants. Dashed lines indicate the average of linear fits to accuracy and RTs along parametric variations of acoustic detail and predictiveness. Note the different scaling of $y$-axes. $\boldsymbol{B}$, Bars represent average linear coefficients quantifying the increase in accuracy and decrease in RTs with each level of acoustic detail or predictiveness for younger (black) and older (magenta) participants. The age group $\times$ stimulus dimension (acoustic detail vs predictiveness) interaction approached significance for accuracy measures $(p=0.076)$ and reached significance for RTs $(p=0.022)$. Error bars indicate \pm 1 SEM.

\section{Results}

Acoustic detail and predictiveness enhance performance

Figure $2 A$ shows mean accuracy and response times in the number comparison task as a function of acoustic detail and predictiveness. Parametric variations along either acoustic detail or predictiveness were quantified as the estimated coefficient from linear fits to accuracy and response times. Testing the linear coefficients against zero revealed that, with higher levels of acoustic detail, accuracy increased $\left(t_{37}=17.81 ; p<0.001 ; r=0.95\right)$ and response time decreased $\left(t_{37}=-6.95 ; p<0.001 ; r=0.75\right)$. Similarly, for higher levels of predictiveness, accuracy increased $\left(t_{37}=5.92 ; p<0.001 ; r=0.70\right)$ and response time decreased $\left(t_{37}\right.$ $=-5.31 ; p<0.001 ; r=0.66)$. The difference in overall accuracy between age groups approached significance $\left(t_{36}=1.95 ; p=\right.$ $0.059 ; r=0.31$ ), indicating a slightly higher overall task accuracy for older participants. Overall response times relative to $\mathrm{S} 2$ onset did not differ between age groups (mean response time younger: 2.28 s, older: $\left.2.23 \mathrm{~s} ; t_{36}=0.28 ; p=0.783 ; r=0.05\right)$.

Differential effects of stimulus dimension (acoustic detail vs predictiveness) and age group (younger vs older) on linear coefficients for accuracy and response times were analyzed using repeated-measures ANOVAs (within-subject factor: stimulus dimension; between-subject factor: age group). The main effect of stimulus dimension was significant for accuracy $\left(F_{(1,36)}=\right.$ $\left.117.88 ; p<0.001, \eta_{p}^{2}=0.77\right)$ and for response times $\left(F_{(1,36)}=\right.$ $22.38 ; p<0.001, \eta_{p}^{2}=0.38$ ), showing that the modulation of performance (i.e., accuracy increase and response time decrease) was stronger for acoustic detail than for predictiveness (Fig. 2B). There was no significant main effect of age group on linear coefficients for accuracy $\left(F_{(1,36)}=0.14 ; p=0.714, \eta_{p}^{2}=0.004\right)$ but on linear coefficients for response times $\left(F_{(1,36)}=4.52 ; p=0.040\right.$, $\left.\eta_{p}^{2}=0.11\right)$, indicating that the decrease in response times was stronger in older than younger participants, regardless of the stimulus dimension.

Critically, the age group $\times$ stimulus dimension interaction on linear coefficients for accuracy approached statistical significance $\left(F_{(1,36)}=3.34 ; p=0.076, \eta_{p}^{2}=0.09\right)$ and reached statistical significance for linear coefficients for response times $\left(F_{(1,36)}=\right.$ $\left.5.69 ; p=0.022, \eta_{p}^{2}=0.14\right)$. Figure $2 B$ indicates the direction of these interactions. Older compared with younger participants' accuracy and response times were affected more strongly with more acoustic detail, whereas predictiveness diminished (for response times) or reversed (for accuracy) this age difference. Post hoc tests for age effects revealed that the linear coefficients quantifying changes in response times with acoustic detail were significantly smaller for older compared with younger participants $\left(t_{36}=2.53\right.$; $p=0.016 ; r=0.39$ ). All remaining pairwise comparisons did not reach statistical significance (all $p>0.15$; all $r<0.22$ ).

\section{Temporal dynamics of alpha oscillations}

Before testing effects of varying acoustic detail and stimulus predictiveness on alpha power, we analyzed temporal dynamics of overall alpha power $(7-13 \mathrm{~Hz})$ across all trials, that is, independent of experimental conditions. Alpha power was enhanced during acoustic stimulation $(0-4.25 \mathrm{~s})$, before returning to baseline at the end of the trial (Fig. $3 A$ ). The increase in alpha power was strongest at parietal electrode sites. We tested whether the time course of overall alpha power (averaged across five parietal electrodes) differed between age groups (Fig. 3B). Alpha power was lower in older than younger participants only toward the end of the trial $(>3.9 \mathrm{~s}$; multiple independent-samples $t$ tests for 20 ms time intervals; $p<0.05$, FDR-corrected; $R=0.51$ ).

\section{Acoustic detail and predictiveness modulate alpha power}

Figure $4 A$ shows the effects of increasing acoustic detail and predictiveness on alpha power $(7-13 \mathrm{~Hz})$. Both effects were quantified by linear coefficients (slopes) reflecting the change in alpha power with increasing levels of acoustic detail or with increasing levels of predictiveness. Cluster-based permutation tests revealed two significant clusters for the effect of acoustic detail, referred to as A1 $(p=0.006 ; R=0.41 ; 0.82-1.92 \mathrm{~s})$ and $\mathrm{A} 2(p<0.001 ; R=$ $0.46 ; 3.52-4.88 \mathrm{~s})$. Similarly, two significant clusters were found for the effect of predictiveness, referred to as P1 $(p<0.001 ; R=$ $0.41 ; 1.22-2.60 \mathrm{~s})$ and $\mathrm{P} 2(p=0.015 ; R=0.39 ; 2.64-3.68 \mathrm{~s})$. No significant positive clusters were observed. For the significant negative clusters, linear coefficients were significantly $<0$. That 
A Grand-average oscillatory power

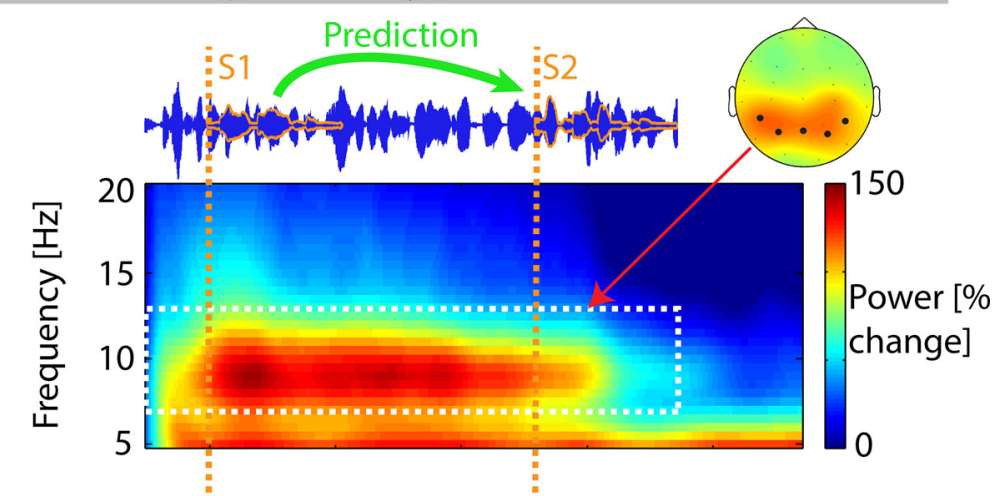

B Temporal dynamics of alpha $(7-13 \mathrm{~Hz})$ power

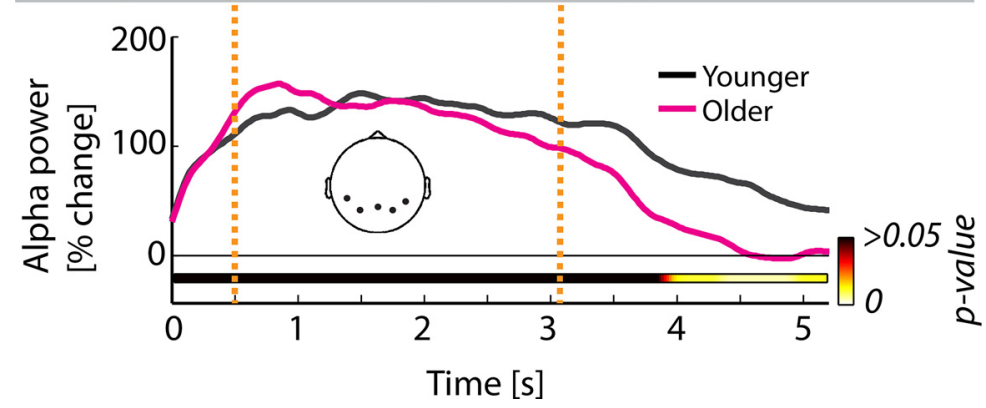

Figure 3. Overall temporal dynamics of alpha power. $\boldsymbol{A}$, Grand average overall oscillatory power (averaged across both age groups and all scalp electrodes) during the auditory number comparison task. Topographical map shows alpha power $(7-13 \mathrm{~Hz})$ during acoustic stimulation $(0-4.25 \mathrm{~s})$. Electrodes exhibiting strongest alpha power are highlighted. $\boldsymbol{B}$, Time courses of mean alpha power (averaged across five parietal electrodes) for younger (black) and older (magenta) participants. $p$ values for the comparison of alpha power between age groups (multiple independent-samples $t$ tests for 20 ms time intervals; FDR-corrected) in horizontal bar indicate lower alpha power for older participants in the end of the trial (>3.9s).

is, alpha power decreased with increasing acoustic detail (higher temporal fine structure cutoffs) and increasing predictiveness (higher absolute numerical difference between S1 and 60). For all significant clusters, these alpha power decreases were significant at a large number of electrodes (Fig. $4 B$, topographic maps): Clusters A1, A2, and P1 spanned 26 of 28 scalp electrodes; cluster P2 spanned 20 of 28 scalp electrodes.

Critically, the temporal occurrence of significant clusters matched precisely with the manipulations of acoustic detail and predictiveness. That is, alpha power decreased during and shortly after spoken digits ( $\mathrm{S} 1$ and $\mathrm{S} 2$ ) when more acoustic detail facilitated the encoding of digits (clusters A1 and A2). When S1 was better predictive of $\mathrm{S} 2$, alpha power decreased significantly during the time period when $\mathrm{S} 2$ could be predicted, that is, between the presentation of S1 and S2 (clusters P1 and P2).

To test whether the effects of acoustic detail and predictiveness on alpha power interact, power estimates for the conjunction of clusters in time-frequency-electrode space were submitted to repeatedmeasures ANOVAs (factors: acoustic detail, predictiveness). For the two conjunctions of clusters in the present data $(\mathrm{A} 1 \cap \mathrm{P} 1, \mathrm{~A} 2 \cap \mathrm{P} 2)$, neither the two-way interaction acoustic detail $\times$ predictiveness nor the three-way interaction with age group was significant (all $p>0.3$, all $\eta_{p}^{2} \leq 0.03$ ), thus indicating independent influences of acoustic detail and predictiveness on alpha power.

The effect of age group on linear coefficients in the four significant clusters was tested with a repeated-measures ANOVA (within-subject factor: cluster; between-subject factor: age group). Modeling the four clusters within one factor acknowledges that the clusters were temporally independent as they occurred in distinct (only partly overlapping) time intervals. The main effects of cluster $\left(F_{(3,108)}=1.13 ; p=0.34, \eta_{p}^{2}=\right.$ 0.03 ; no significant violation of sphericity: Mauchly's test, $p=0.26$ ) and age group $\left(F_{(1,36)}=0.08 ; p=0.782, \eta_{p}^{2}<0.01\right)$ were not significant. However, the age group $X$ cluster interaction was significant $\left(F_{(3,108)}\right.$ $=6.58 ; p<0.001, \eta_{p}^{2}=0.16$ ), indicating a different pattern of alpha power modulations in the four clusters for younger compared with older participants (Fig. $4 B$; note that this interaction was also significant using overall alpha power in the four clusters and the linear decrease in overall alpha power during the trial as covariates; $p=0.047$ and $p<0.001$, respectively). Post hoc tests for differences between age groups in the four clusters revealed a significant effect of age group on linear coefficients in the A1 cluster $\left(t_{36}=2.17 ; p=\right.$ $0.036 ; r=0.34$, uncorrected), but not in the three remaining clusters (A2, P1, P2; all $p>0.15$; all $r<0.24$ ). That is, older participants' alpha power during the encoding of S1 decreased stronger with increasing acoustic detail compared with younger participants.

Corroborating this age difference, the significant A1 cluster was found only for the group of older participants when the cluster analysis was performed separately for the two age groups. This finding was well in line with behavioral results (Fig. 2) where varying acoustic detail also had a relative stronger impact on older participants' task performance.

\section{Alpha oscillations predict subjective measures of difficulty}

An important question of the present study was whether participants' self-rated difficulty of speech-in-noise listening and their confidence in the numerical comparison were related to fluctuations in alpha power. We investigated this question with respect to subjective ratings of listening effort (self-rated after the experiment) and confidence ratings in the end of each experimental trial.

The mean estimated linear coefficients (reflecting modulation of alpha power by acoustic detail and predictiveness) across all clusters (Fig. 4) were correlated with participants' self-reported effort of listening to a single speaker in the presence of background noise (Fig. 5A). We observed a significant positive correlation $(r=0.484 ; p=0.002 ; \mathrm{df}=36)$, indicating that listeners who experienced higher subjective listening effort exhibited weaker alpha power modulations with varying acoustic detail and predictiveness. The correlation was also significant when calculated for both age groups separately (younger: $r=0.54$; $p=$ 0.021; df $=16$; older: $r=0.48 ; p=0.032 ; \mathrm{df}=18$ ).

We tested whether alpha power would correlate with confidence ratings independent of variations in acoustic detail and predictiveness. To this end, mean alpha power $(7-13 \mathrm{~Hz})$ for single trials in the time period from the onset of the earliest significant cluster (A1, $0.82 \mathrm{~s}$ ) until the offset of the latest significant cluster (A2, $4.88 \mathrm{~s}$ ) at all scalp electrodes was determined. Singletrial confidence ratings within each factor combination in the 6 (acoustic detail) $\times 6$ (predictiveness) design were binned into 

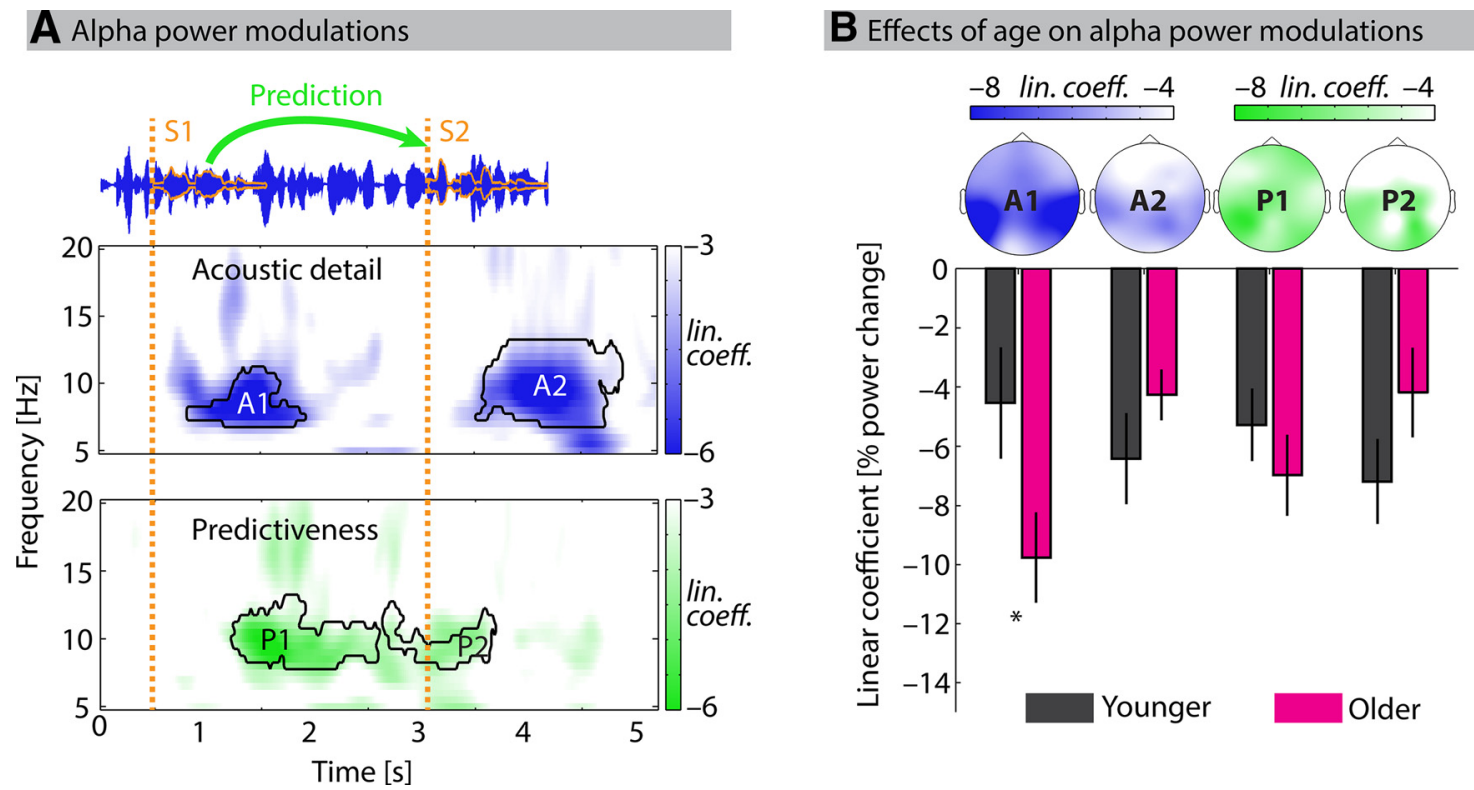

Figure 4. Effects of acoustic detail and predictiveness on alpha power. A, Alpha power $(7-13 \mathrm{~Hz})$ decreased significantly with higher levels of acoustic detail (top: clusters A1 and A2) and higher levels of predictiveness (bottom: clusters P1 and P2) in distinct time periods. Estimated linear coefficients indicate the relative change in alpha power (in percentage) with each level of acoustic detail or predictiveness. $\boldsymbol{B}$, Topographical maps of clusters show a global decrease of alpha power with acoustic detail and predictiveness with the largest power decrease over centroparietal electrode sites. Bars represent average linear coefficients for younger (black) and older (magenta) participants. Significantly smaller linear coefficients for older participants in cluster A1 indicated a stronger alpha power modulation as a function of acoustic detail for older listeners approximately at $\mathrm{S} 1$ offset ( $p=0.036$, uncorrected). Error bars indicate \pm 1 SEM. ${ }^{*} p<0.05$.
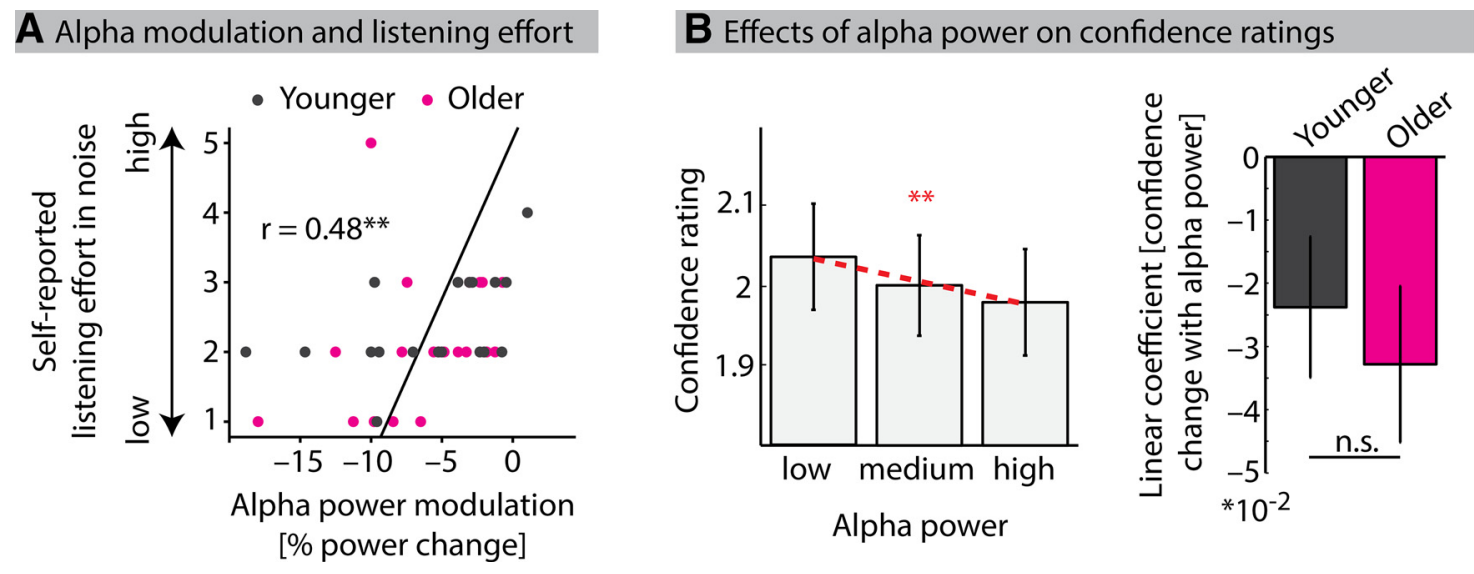

Figure 5. Alpha power relates to subjective difficulty measures. $A$, Alpha power modulation (reflecting mean linear coefficients of alpha power changes with each level of acoustic detail and predictiveness across four significant clusters) as a function of participants' self-reported subjective listening effort in background noise (significant Spearman correlation, $p=0.002$ ). Data for younger and older participants are shown in black and magenta, respectively. $\boldsymbol{B}$, Left, Bars represent mean confidence ratings for trials with low, medium, and high alpha power. Between-condition effects were eliminated by binning trials according to alpha power separately for each factor combination of acoustic detail and predictiveness. Red dashed line indicates the mean linear fit to confidence ratings. Confidence ratings decreased with higher alpha power $(p=0.001)$. Right, Bars represent the average linear coefficients (quantifying changes in confidence ratings with each level of alpha power) for younger (black) and older (magenta) participants. Error bars indicate \pm 1 SEM. ${ }^{* *} p<0.01$. n.s., Not significant.

three alpha power percentiles (no overlap; low, medium, and high alpha power). Next, single-trial confidence ratings were averaged within each bin, and then across the $6 \times 6$ levels, resulting in three values for each participant, reflecting confidence in trials with low, medium, and high alpha power (Fig. 5B). The coefficients from linear fits to changes in confidence ratings over these three levels of alpha power were significantly $<0\left(t_{37}=-3.44 ; p=0.001\right.$; $r=0.50)$. That is, confidence ratings were higher in trials with lower alpha power. Linear coefficients did not differ significantly between age groups $\left(t_{36}=0.54 ; p=0.59 ; r=0.09\right.$; Fig. $5 B$, right $)$.

\section{Discussion}

We tested whether alpha oscillations track changing task demands in a multitalker situation in younger and older listeners.
Results can be summarized as follows: (1) Alpha power decreased with increasing acoustic detail and, critically, also with increasing stimulus predictiveness. (2) In older participants, increased acoustic detail induced a stronger behavioral benefit and a stronger alpha power decrease. (3) Stronger alpha power modulations with acoustic detail and predictiveness, as well as lower overall alpha power, predicted lower subjective difficulty.

\section{Listening demands modulate alpha oscillatory power}

Behavioral results show that accuracy in a two-talker auditory number comparison task increased with more acoustic detail (temporal fine structure) in the stimulus materials and also with better numerical predictiveness (Fig. 2). This agrees with previous research showing that: (1) preserved temporal fine structure 
facilitates perceptual segregation of competing talkers (e.g., Hopkins et al., 2008; Hopkins and Moore, 2010; Lunner et al., 2012); and (2) numerical predictiveness improves stimulus comparison (e.g., Scheibe et al., 2010). We extend previous observations by relating manipulations of acoustic detail and predictiveness to neural alpha oscillations.

On the neurophysiological level, alpha power decreased in distinct time intervals with parametric variations along two stimulus dimensions: First, with increasing acoustic detail, alpha power decreased during the encoding of target digits (Fig. 4). This is consistent with previous observations of reduced alpha power for less degraded speech materials (Obleser et al., 2012; e.g., Obleser and Weisz, 2012). Although acoustic detail was manipulated during the entire trial, alpha power modulation occurred exclusively during the encoding of task-relevant digits. This suggests that the modulation of alpha power with acoustic detail is guided by attention to target signals.

Second, with better stimulus predictiveness, alpha power decreased during the prediction of the second digit (i.e., between the two digits). Although alpha power modulations have been found for varying temporal predictions of "when" a target stimulus would occur (e.g., Rohenkohl and Nobre, 2011; Wilsch et al., 2014), evidence for the prediction of "what" the target stimulus will be have so far been rare (for review, see Arnal and Giraud, 2012). Thus, in the current study, we show that alpha power modulations reflect the predictiveness of upcoming semantic content. Stimulus predictiveness is a complementary source of information (separate from acoustic detail) that listeners can use to reduce the uncertainty in the numerical comparison. In highly predictive trials, participants gather information in favor of a "smaller" or "larger" decision already with the first digit. Thus, increasing decision certainty surfaced as a relative reduction in alpha power and reduced listening demands.

Good performance in our number comparison task required selective attention to digits while ignoring the irrelevant speech masker ("cocktail party problem") (Cherry, 1953). Enhanced alpha power at parieto-occipital sites when attention is directed toward the auditory modality is an established observation (Adrian, 1944; Foxe et al., 1998; Mazaheri et al., 2014). Based on previous localizations of alpha power effects in auditory tasks (Obleser and Weisz, 2012; Obleser et al., 2012), the current parietal distributions of alpha power likely originate from parietal cortex, which belongs to the "dorsal attention network" (Sadaghiani et al., 2010). Increased task difficulty (less acoustic detail or predictiveness) requires more attention to the auditory sensory input. Thus, task-irrelevant sensory modalities (e.g., vision) and task-irrelevant brain processes might be inhibited. Inhibition is likely reflected by enhanced alpha oscillations in a parietal network, which interacts with sensory areas during attention (Banerjee et al., 2011).

\section{Age-related changes in listening behavior and alpha power dynamics}

Overall alpha power was prominently enhanced during the number comparison task (see also Spitzer et al., 2014) but was reduced toward the end of a trial in older participants (Fig. 3B). Critically, overall response times did not differ between age groups, and the stronger alpha power reduction at trial ending for older participants was specific to the alpha frequency band (i.e., no motor-associated beta-band effect in a post hoc analysis). As a consequence, the stronger alpha power decrease toward the end of a trial in older listeners was unlikely driven by an earlier response preparation. Instead, the reduced overall alpha power might reflect decreased maintenance of selective attention in older listeners (Gazzaley et al., 2005). In line with this view, decreased lateralization of alpha power in older participants under high cognitive load has been interpreted as less efficient sustained inhibition of task-irrelevant neural processing (Sander et al., 2012).

In the behavioral results, we found that varying acoustic detail exerted a stronger relative impact on accuracy and response times in older adults. Thus, despite previous reports on reduced sensitivity to temporal fine structure variations in older adults (Grose and Mamo, 2010; Hopkins and Moore, 2011; Moore et al., 2012), older listeners in the current study relied relatively more on acoustic cues for their performance. The strong dependence on acoustic cues in older listeners is in line with a stronger acousticsdriven decrease in alpha power after the presentation of the first digit in older participants (see also Sebastian et al., 2011). One attractive interpretation is that older listeners' attentional focus is more strongly affected by acoustic features of the external signal, potentially related to their difficulty in ignoring irrelevant auditory distractors (Chao and Knight, 1997; Tun et al., 2002; Passow et al., 2014). To our knowledge, there has been only one (behavioral) study that has shown a stronger dependence of speech recognition on spectral degradations at an older age, comparable with our observation (Schvartz et al., 2008). The present results thus demonstrate that age-related changes in listening behavior are reflected in neural alpha oscillations.

Notably, one rationale in the current study was to equalize audibility of materials (through individual control for frequencyspecific audiometric thresholds) and the overall performance level (through individual adjustment of the digit-to-masker ratio) across participants to avoid propagated effects of hearing acuity on brain dynamics (Tremblay et al., 2003; Peelle et al., 2011). However, conventional auditory threshold measures do not capture all aspects of auditory processing acuity. For instance, age and noise exposure might affect the neural encoding of suprathreshold sounds (Kujawa and Liberman, 2009; Ruggles et al., 2012; Furman et al., 2013) and could also contribute to observed age differences in listening behavior and alpha dynamics. Although sensory encoding is commonly impeded in older listeners, it is unclear whether this affects perception (Clinard et al., 2010; but see also Ruggles et al., 2012) and electrophysiological measures of cortical activity.

\section{Alpha oscillations relate to subjective difficulty}

We here extend previous findings of alpha oscillations as a neural marker of cognitive effort (e.g., Klimesch, 1999; Jensen et al., 2002) to one of the most common communication situations, that is, comprehending speech in multitalker situations. Participants who showed weaker alpha power modulations with varying task difficulty reported higher difficulties of listening to speech in noise (Fig. 5A). This is compatible with the view that higher neural variability accompanies enhanced behavioral performance (Garrett et al., 2011; Erb and Obleser, 2013).

In addition to interindividual differences in alpha power, we also found that intraindividual, trial-to-trial variations in alpha power affected post-trial confidence ratings: Lower alpha power during a trial predicted higher confidence of listeners in their own decision (Fig. 5B). Although correlations between alpha power and behavior have been found previously (e.g., Klimesch et al., 1997; Haegens et al., 2011; Wilsch et al., 2014), the present changes in alpha power exerted an impact on subjective confidence ratings, a measure of so-called meta-cognition (Shea et al., 2014). Thus, fluctuations in alpha power not only reflect changes 
in the external stimulation, but they also constitute a change in brain state, which is independent of the stimulation yet can impact behavior (Obleser and Weisz, 2012). The direction of the observed effect (lower alpha power for higher confidence ratings) supports the view that decreased alpha power reflects reduced task demands. These observations significantly extend the current understanding of alpha oscillations as a marker of subjective difficulty during effortful listening.

In conclusion, the current study shows that alpha oscillations support auditory processing in younger and older listeners in noisy environments in multiple ways. First, alpha oscillations are modulated by stimulation-related encoding demands induced by acoustic detail but are also sensitive to the degree of stimulus predictiveness. Second, task performance and alpha modulation in older listeners are stronger affected by varying acoustic detail. This speaks to changes in attentional control at an older age. Last, alpha oscillatory dynamics explain interindividual and intraindividual differences in introspective task demand. In sum, alpha dynamics are a promising neural marker to elucidate on individual and age-related difficulties in sensation, perception, as well as decision-making.

\section{References}

Adrian ED (1944) Brain rhythms. Nature 153:360-362. CrossRef

Arnal LH, Giraud AL (2012) Cortical oscillations and sensory predictions. Trends Cogn Sci 16:390-398. CrossRef Medline

Attneave F, Olson RK (1971) Pitch as a medium: a new approach to psychophysical scaling. Am J Psychol 84:147-166. CrossRef Medline

Banerjee S, Snyder AC, Molholm S, Foxe JJ (2011) Oscillatory alpha-band mechanisms and the deployment of spatial attention to anticipated auditory and visual target locations: supramodal or sensory-specific control mechanisms? J Neurosci 31:9923-9932. CrossRef Medline

Becker R, Pefkou M, Michel CM, Hervais-Adelman AG (2013) Left temporal alpha-band activity reflects single word intelligibility. Front Syst Neurosci 7:121. CrossRef Medline

Benjamini Y, Hochberg Y (1995) Controlling the false discovery rate: a practical and powerful approach to multiple testing. J R Stat Soc B 57: 289-300.

Bonnefond M, Jensen O (2012) Alpha oscillations serve to protect working memory maintenance against anticipated distracters. Curr Biol 22:19691974. CrossRef Medline

British Society of Audiology (2011) Recommended Procedure: Pure-tone air-conduction and bone-conduction threshold audiometry with and without masking. Berkshire, UK: British Society of Audiology.

Chao LL, Knight RT (1997) Prefrontal deficits in attention and inhibitory control with aging. Cereb Cortex 7:63-69. CrossRef Medline

Cherry CE (1953) Some experiments on the recognition of speech, with one and with two ears. J Acoust Soc Am 25:975-979. CrossRef

Clinard CG, Tremblay KL, Krishnan AR (2010) Aging alters the perception and physiological representation of frequency: evidence from human frequency-following response recordings. Hear Res 264:48-55. CrossRef Medline

Desimone R, Duncan J (1995) Neural mechanisms of selective visual attention. Annu Rev Neurosci 18:193-222. CrossRef Medline

Doppelmayr MM, Klimesch W, Pachinger T, Ripper B (1998) The functional significance of absolute power with respect to event-related desynchronization. Brain Topogr 11:133-140. CrossRef Medline

Erb J, Obleser J (2013) Upregulation of cognitive control networks in older adults' speech comprehension. Front Syst Neurosci 7:116. CrossRef Medline

Foxe JJ, Simpson GV, Ahlfors SP (1998) Parieto-occipital approximately 10 $\mathrm{Hz}$ activity reflects anticipatory state of visual attention mechanisms. Neuroreport 9:3929-3933. CrossRef Medline

Furman AC, Kujawa SG, Liberman MC (2013) Noise-induced cochlear neuropathy is selective for fibers with low spontaneous rates. J Neurophysiol 110:577-586. CrossRef Medline

Garrett DD, Kovacevic N, McIntosh AR, Grady CL (2011) The importance of being variable. J Neurosci 31:4496-4503. CrossRef Medline

Gazzaley A, Cooney JW, Rissman J, D’Esposito M (2005) Top-down sup- pression deficit underlies working memory impairment in normal aging. Nat Neurosci 8:1298-1300. CrossRef Medline

Grose JH, Mamo SK (2010) Processing of temporal fine structure as a function of age. Ear Hear 31:755-760. CrossRef Medline

Haegens S, Händel BF, Jensen O (2011) Top-down controlled alpha band activity in somatosensory areas determines behavioral performance in a discrimination task. J Neurosci 31:5197-5204. CrossRef Medline

Herrmann B, Henry MJ, Scharinger M, Obleser J (2014) Supplementary motor area activations predict individual differences in temporal-change sensitivity and its illusory distortions. Neuroimage 101:370-379. CrossRef Medline

Hopkins K, Moore BC (2010) The importance of temporal fine structure information in speech at different spectral regions for normal-hearing and hearing-impaired subjects. J Acoust Soc Am 127:1595-1608. CrossRef Medline

Hopkins K, Moore BC (2011) The effects of age and cochlear hearing loss on temporal fine structure sensitivity, frequency selectivity, and speech reception in noise. J Acoust Soc Am 130:334-349. CrossRef Medline

Hopkins K, Moore BC, Stone MA (2008) Effects of moderate cochlear hearing loss on the ability to benefit from temporal fine structure information in speech. J Acoust Soc Am 123:1140-1153. CrossRef Medline

Jensen O, Mazaheri A (2010) Shaping functional architecture by oscillatory alpha activity: gating by inhibition. Front Hum Neurosci 4:186. CrossRef Medline

Jensen O, Gelfand J, Kounios J, Lisman JE (2002) Oscillations in the alpha band $(9-12 \mathrm{~Hz})$ increase with memory load during retention in a shortterm memory task. Cereb Cortex 12:877-882. CrossRef Medline

Kerlin JR, Shahin AJ, Miller LM (2010) Attentional gain control of ongoing cortical speech representations in a "cocktail party." J Neurosci 30:620628. CrossRef

Kitayama S (1991) Impairment of perception by positive and negative affect. Cogn Emotion 5:255-274. CrossRef

Klimesch W (1999) EEG alpha and theta oscillations reflect cognitive and memory performance: a review and analysis. Brain Res Brain Res Rev 29:169-195. CrossRef Medline

Klimesch W, Doppelmayr M, Pachinger T, Ripper B (1997) Brain oscillations and human memory: EEG correlates in the upper alpha and theta band. Neurosci Lett 238:9-12. CrossRef Medline

Klimesch W, Sauseng P, Gerloff C (2003) Enhancing cognitive performance with repetitive transcranial magnetic stimulation at human individual alpha frequency. Eur J Neurosci 17:1129-1133. CrossRef Medline

Kujawa SG, Liberman MC (2009) Adding insult to injury: cochlear nerve degeneration after "temporary" noise-induced hearing loss. J Neurosci 29:14077-14085. CrossRef Medline

Levitt H (1971) Transformed up-down methods in psychoacoustics. J Acoust Soc Am 49 [Suppl 2]:467.

Lunner T, Hietkamp RK, Andersen MR, Hopkins K, Moore BC (2012) Effect of speech material on the benefit of temporal fine structure information in speech for young normal-hearing and older hearing-impaired participants. Ear Hear 33:377-388. CrossRef Medline

Maris E, Oostenveld R (2007) Nonparametric statistical testing of EEG- and MEG-data. J Neurosci Methods 164:177-190. CrossRef Medline

Mazaheri A, van Schouwenburg MR, Dimitrijevic A, Denys D, Cools R, Jensen O (2014) Region-specific modulations in oscillatory alpha activity serve to facilitate processing in the visual and auditory modalities. Neuroimage 87:356-362. CrossRef Medline

Meister H, Schreitmuller S, Grugel L, Ortmann M, Beutner D, Walger M, Meister IG (2012) Cognitive resources related to speech recognition with a competing talker in young and older listeners. Neuroscience 232C: 74-82. CrossRef Medline

Meyer L, Obleser J, Friederici AD (2013) Left parietal alpha enhancement during working memory-intensive sentence processing. Cortex 49:711721. CrossRef Medline

Moore BC (2008) The role of temporal fine structure processing in pitch perception, masking, and speech perception for normal-hearing and hearing-impaired people. J Assoc Res Otolaryngol 9:399-406. CrossRef Medline

Moore BC, Alcántara JI, Glasberg BR (1998) Development and evaluation of a procedure for fitting multi-channel compression hearing aids. Br J Audiol 32:177-195. CrossRef Medline

Moore BC, Glasberg BR, Stoev M, Füllgrabe C, Hopkins K (2012) The influence of age and high-frequency hearing loss on sensitivity to temporal 
fine structure at low frequencies (L). J Acoust Soc Am 131:1003-1006. CrossRef Medline

Moyer RS, Landauer TK (1967) Time required for judgements of numerical inequality. Nature 215:1519-1520. CrossRef Medline

Obleser J, Weisz N (2012) Suppressed alpha oscillations predict intelligibility of speech and its acoustic details. Cereb Cortex 22:2466-2477. CrossRef Medline

Obleser J, Wise RJ, Alex Dresner MA, Scott SK (2007) Functional integration across brain regions improves speech perception under adverse listening conditions. J Neurosci 27:2283-2289. CrossRef Medline

Obleser J, Eisner F, Kotz SA (2008) Bilateral speech comprehension reflects differential sensitivity to spectral and temporal features. J Neurosci 28: 8116-8123. CrossRef Medline

Obleser J, Wöstmann M, Hellbernd N, Wilsch A, Maess B (2012) Adverse listening conditions and memory load drive a common alpha oscillatory network. J Neurosci 32:12376-12383. CrossRef Medline

Oostenveld R, Fries P, Maris E, Schoffelen JM (2011) FieldTrip: open source software for advanced analysis of MEG, EEG, and invasive electrophysiological data. Comput Intell Neurosci 2011:156869. CrossRef Medline

Passow S, Westerhausen R, Hugdahl K, Wartenburger I, Heekeren HR, Lindenberger U, Li SC (2014) Electrophysiological correlates of adult age differences in attentional control of auditory processing. Cereb Cortex 24:249-260. CrossRef Medline

Peelle JE, Troiani V, Grossman M, Wingfield A (2011) Hearing loss in older adults affects neural systems supporting speech comprehension. J Neurosci 31:12638-12643. CrossRef Medline

Pichora-Fuller MK, Souza PE (2003) Effects of aging on auditory processing of speech. Int J Audiol 42[Suppl 2]:2S11-S16.

Pichora-Fuller MK, Schneider BA, Daneman M (1995) How young and old adults listen to and remember speech in noise. J Acoust Soc Am 97:593608. CrossRef Medline

Rohenkohl G, Nobre AC (2011) Alpha oscillations related to anticipatory attention follow temporal expectations. J Neurosci 31:14076-14084. CrossRef Medline

Rosenthal R (1994) Parametric measures of effect size. In: Handbook of research synthesis (Cooper H, Hedges LV, eds), pp 231-244. New York: Russell Sage Foundation.

Ruggles D, Bharadwaj H, Shinn-Cunningham BG (2012) Why middle-aged listeners have trouble hearing in everyday settings. Curr Biol 22:14171422. CrossRef Medline

Sadaghiani S, Scheeringa R, Lehongre K, Morillon B, Giraud AL, Kleinschmidt A (2010) Intrinsic connectivity networks, alpha oscillations, and tonic alertness: a simultaneous electroencephalography/functional magnetic resonance imaging study. J Neurosci 30:10243-10250. CrossRef Medline

Sander MC, Werkle-Bergner M, Lindenberger U (2012) Amplitude modulations and inter-trial phase stability of alpha-oscillations differentially reflect working memory constraints across the lifespan. Neuroimage 59: 646-654. CrossRef Medline

Sauseng P, Klimesch W, Heise KF, Gruber WR, Holz E, Karim AA, Glennon M, Gerloff C, Birbaumer N, Hummel FC (2009) Brain oscillatory substrates of visual short-term memory capacity. Curr Biol 19:1846-1852. CrossRef Medline
Scheibe C, Ullsperger M, Sommer W, Heekeren HR (2010) Effects of parametrical and trial-to-trial variation in prior probability processing revealed by simultaneous electroencephalogram/functional magnetic resonance imaging. J Neurosci 30:16709-16717. CrossRef Medline

Schvartz KC, Chatterjee M, Gordon-Salant S (2008) Recognition of spectrally degraded phonemes by younger, middle-aged, and older normalhearing listeners. J Acoust Soc Am 124:3972-3988. CrossRef Medline

Sebastián M, Reales JM, Ballesteros S (2011) Ageing affects event-related potentials and brain oscillations: a behavioral and electrophysiological study using a haptic recognition memory task. Neuropsychologia 49: 3967-3980. CrossRef Medline

Shamma S, Lorenzi C (2013) On the balance of envelope and temporal fine structure in the encoding of speech in the early auditory system. J Acoust Soc Am 133:2818-2833. CrossRef Medline

Shannon RV, Zeng FG, Kamath V, Wygonski J, Ekelid M (1995) Speech recognition with primarily temporal cues. Science 270:303-304. CrossRef Medline

Shea N, Boldt A, Bang D, Yeung N, Heyes C, Frith CD (2014) Suprapersonal cognitive control and metacognition. Trends Cogn Sci 18:186193. CrossRef Medline

Slaney M (1993) An efficient implementation of the Patterson-Holdsworth Auditory Filter Bank. Cupertino, CA: Apple Computer.

Smith ZM, Delgutte B, Oxenham AJ (2002) Chimaeric sounds reveal dichotomies in auditory perception. Nature 416:87-90. CrossRef Medline

Spitzer B, Fleck S, Blankenburg F (2014) Parametric alpha- and beta-band signatures of supramodal numerosity information in human working memory. J Neurosci 34:4293-4302. CrossRef Medline

Strauß A, Wöstmann M, Obleser J (2014) Cortical alpha oscillations as a tool for auditory selective inhibition. Front Hum Neurosci 8:350. CrossRef Medline

Tremblay KL, Piskosz M, Souza P (2003) Effects of age and age-related hearing loss on the neural representation of speech cues. Clin Neurophysiol 114:1332-1343. CrossRef Medline

Tun PA, O'Kane G, Wingfield A (2002) Distraction by competing speech in young and older adult listeners. Psychol Aging 17:453-467. CrossRef Medline

van Ede F, de Lange F, Jensen O, Maris E (2011) Orienting attention to an upcoming tactile event involves a spatially and temporally specific modulation of sensorimotor alpha- and beta-band oscillations. J Neurosci 31:2016-2024. CrossRef Medline

Wilsch A, Henry MJ, Herrmann B, Maess B, Obleser J (2014) Alpha oscillatory dynamics index temporal expectation benefits in working memory. Cereb Cortex. Advance online publication. Retrieved Mar 2, 2014. doi: 10.1093/cercor/bhu004. CrossRef Medline

Wingfield A, Tun PA, McCoy S (2005) Hearing loss in older adulthood: what it is and how it interacts with cognitive performance. Curr Dir Psychol Sci 14:144-148. CrossRef

Wöstmann M, Schröger E, Obleser J (2014) Acoustic detail guides attention allocation in a selective listening task. J Cogn Neurosci. Advance online publication. Retrieved Nov 12, 2014. doi: 10.1162/jocn_a_00761. CrossRef Medline 